\title{
SUPPLEMANTARY INFORMATION
}

\author{
Addditional freshwater $\delta^{13} \mathrm{C}$ data in a outdoor pond
}

We also have performed $\delta^{13} \mathrm{C}$ measurements on biofilms in different developmental stages, that have grown in a pond outdoors, which was a part of a $750 \mathrm{~m}$ long helophyte filter used for polishing discharge of waste water to open water systems. The biofilms were grown on clean horizontal placed glass plates at three different depths in the water column in order to create different overall daily irradiances. The biofilms were harvested at different time points (after one week and after two weeks) after inoculation in the water column. This allowed us to sample different developmental stages. The experiment has been performed twice (one week difference in start time). DIC values have been measured and were $\sim 16 \%$. Since we did not follow growth in time we did not measure the light absorbance. Therefore biomass was expressed as wet weight. The data of the different depths have been pooled. We found a similar trend in $\delta^{13} \mathrm{C}$ values with biomass as was found with the development of biofilms in the incubator.

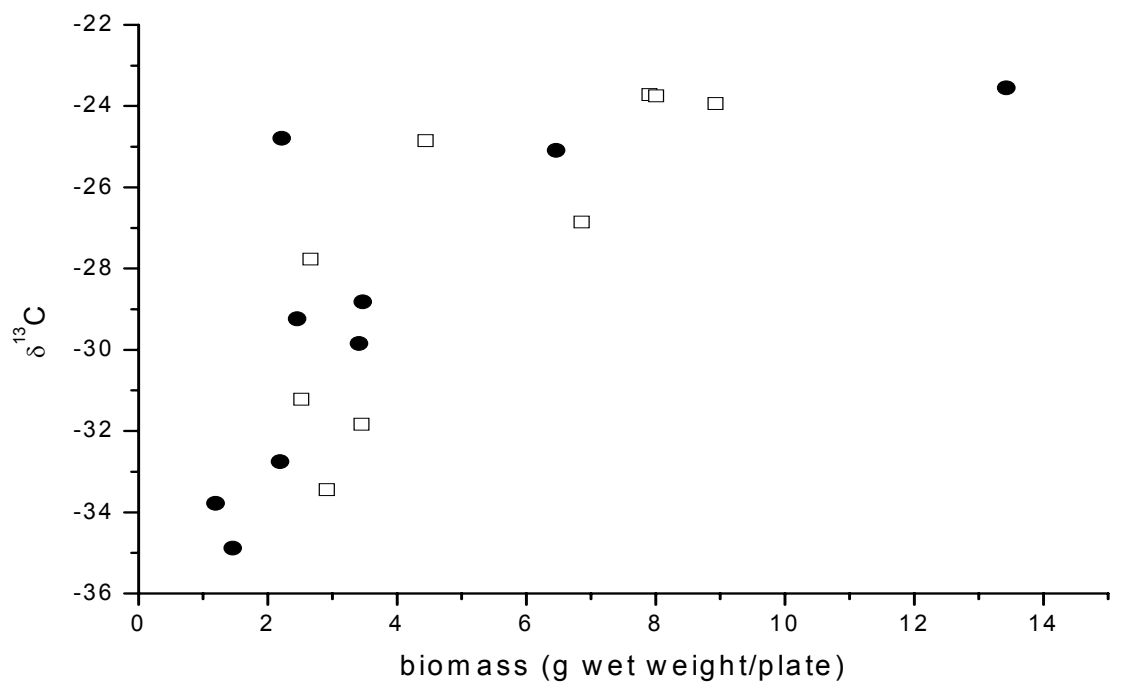

Supplementary Figure 1: Relationship between biomass development and $\delta 13 \mathrm{C}$ value in newly grown biofilms on clean glass plates placed in a helophyte filter. Black circles and open squares indicate two independent measuring series.

Changes in net growth rate during the development of a biofilm.

Theoretically it can be argued that net growth will stop at a certain point due to light limitation. At this point the depth integrated photosynthesis rate will reach a maximum and will be balanced due to an increase in consumption/respiration per $\mathrm{m}^{2}$ due to the thickening of the biofilm. The model presented in Wolf et al, 2007 (which was the same model as used for the ms) also shows that the biofilm cannot exhibit unlimited grow to an infinite thickness. To visualize the decrease in growth rate a graph is included below in which we plot the In value of the biomass versus time (supplementary Figure 2). This graph shows an initial linear part, which shows that growth is exponential in the initial phase, then starts to become less steep, indicating 
a lower growth rate at the end of the exponential phase of the growth curve, and even lower at later stages. As is shown in supplementary Fig. 2 it is possible to estimate the growth rate by taking the first derivative of the In plot at the moment of sampling as an indicator of the growth rate. However, when this method is carried out, the estimate of the growth rate will become somewhat inaccurate, since only few points can be used for the estimates of this line and therefore is prone to short time variation in the incubator due to sloughing etc. We estimated for one run the growth rate in the initial phase (Linear fit in the In graph) by this method, and found exactly the same trend in growth rate as was found with the logistic growth model.

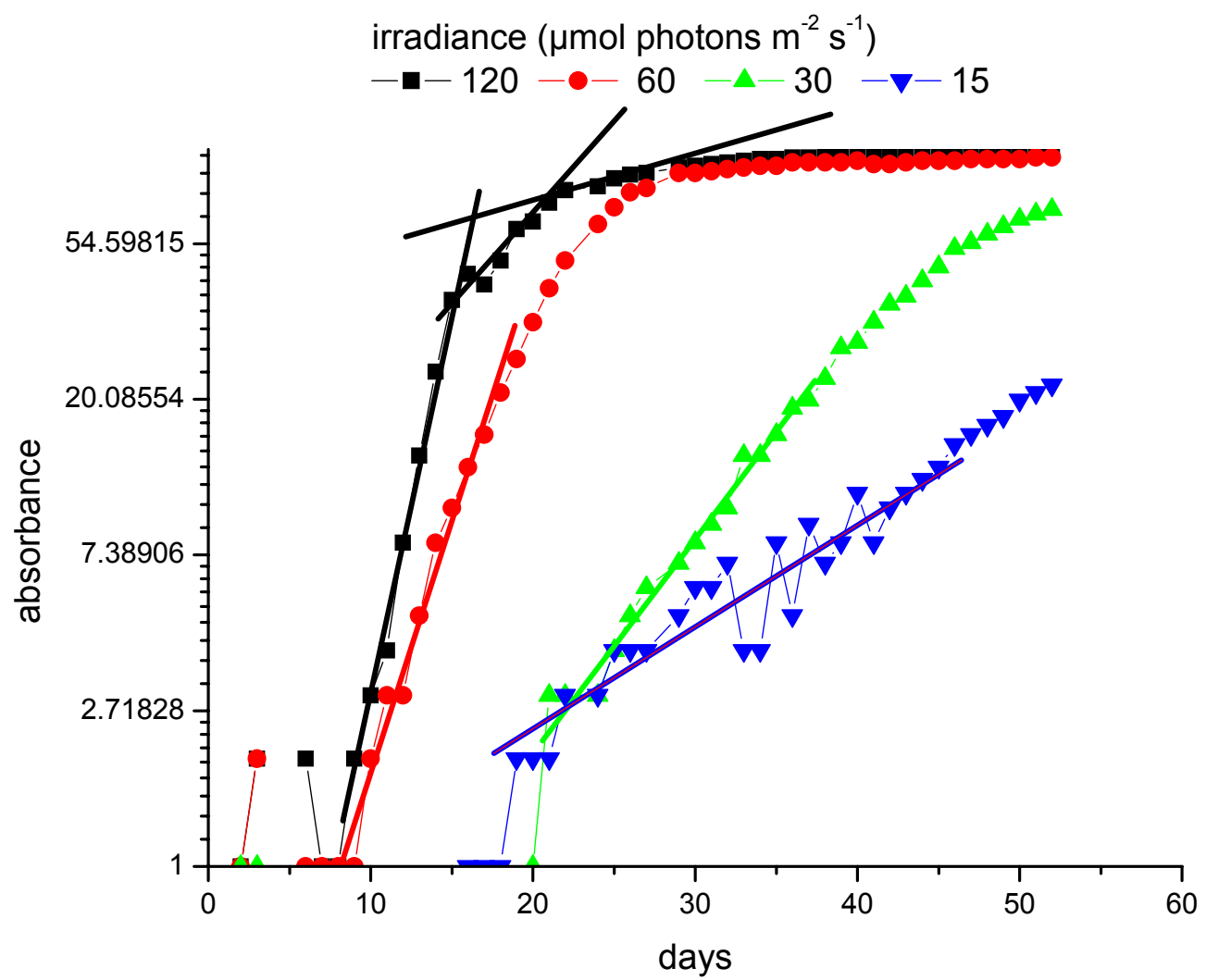

Supplementary Figure 2: Biomass development with time. The biomass is plotted with a In scale in order to fit the growth rate in the initial phase. The angle of the tangent indicates the growth rate. The tangent has been estimated for the initial phase in this graph. In addition some tangent are drawn in the $120 \mu \mathrm{mol}$ photons $\mathrm{m}-2$ $\mathrm{s}-1$ incubation, indicating the decrease in growth rate with the development of the biofilm. 